\title{
Reasarch of Architecture based BP Neural Network in Modeling of Sheave Buffer
}

\author{
$\mathrm{Ke} \mathrm{Bi}^{1, \mathrm{a}}$, Xiang $\mathrm{Li}^{1}$ Zhiying $\mathrm{Tang}^{1}$ Yemeng $\mathrm{Wei}^{1}$ and Gang $\mathrm{Wu}^{1, \mathrm{~b}}$ \\ ${ }^{1}$ Naval University of Engineering, Wuhan 430033, China; \\ abikefly@aliyun.com, bgangwu206@aliyun.com
}

Keywords: Neural network, sheave buffer, modeling, hydraulic system.

\begin{abstract}
In order to solve the problem of non-linear modeling of hydraulic system, a modeling method based on BP neural network is put forward. The complex hydraulic system, according to the structure and relationship of hydraulic system, is divided into simpler subsystems, a complete network is established and performance of network is analysis with the interaction between hydraulic system , a hydraulic buffer system modeling method in MK7-3 slide wheel buffer modeling is discussed, in the application of neural network of the. The results show that the simulation of MK7 type pulley buffer based on BP neural network is accurate.
\end{abstract}

\section{Introduction}

With the increasing weight of carrier based aircraft take-off, improved take-off speed, carrier based aircraft arresting system should also be changed. Stating from the beginning of the MK7-3 type arrester ${ }^{[1]}$, the long run red control device and pulley buffer device and wire end buffer device is add up. Pulley buffer device ,by arresting cable ,series to arresting system, between the carrier based aircraft and arresting system, which mainly reducing the arresting cable vibration, and the arresting of the arresting cable tension peak value in initial period, keeping up the arresting cable tension. Pulley buffer device in aircraft arresting system working environment is complex and is directly related to the arresting cable force and main arresting system, thereby affecting the arresting success rate. The existing research on the pulley buffer is only concentrated in the mathematical model, and cannot describe, predict and optimize it accurately ${ }^{[2,3]}$.

Hydraulic system is a nonlinear system, which can be seen as a number of interacting subsystems, according to structural components and functions. Through researches on subsystem, are combined to form the model of the nonlinear system, with some principles of the subsystem models ${ }^{[4]}$. Based on the structure of the neural network model of the structural characteristics of the hydraulic system, the subsystem decomposition put up into a number of relatively simple subsystems, so every single subsystem is respectively to a neuron subsystem, which namely the establishment of artificial neuron model of each subsystem, the inherent relations between each subsystem is composed of network, the network is nonlinear system model, which is based on the neural network model of the structure of the hydraulic system ${ }^{[5]}$.

\section{Mathematical model of MK7-3 type sheave buffer}

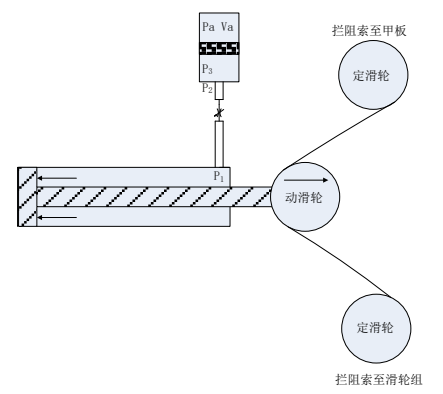

Fig. 1 Structure sketch of MK7-3 sheave buffer 
Device according to the law of impulse, MK7-3 in capture stage role is the block buffer device: $F_{\mathrm{z}} \Delta t=M \Delta v$, the quality of the impact load and the movable pulley is $M$, to pull out of the hydraulic cylinder piston speed is $v_{0}$, hydraulic oil under impactloading pipes into the accumulator, due to the local resistance loss caused by the mutation pipe diameter and pipe running resistance loss, hydraulic cylinder rod cavity pressure $P_{\mathrm{y}}$ increases, the hydraulic oil into the accumulator at the same time, the accumulator in the hydraulic oil volume $V_{c}$ increase, accumulator nitrogen gas cavity $V_{\mathrm{a}}$ is compressed.

According to the work mechanism of the pulley buffer, it can be divided into ${ }^{[6]}$ : the hydraulic cylinder in the impact, the hydraulic cylinder piston rod chamber pressure and the accumulator pressure under the action of moving process; Hydraulic cylinder rod chamber of the hydraulic oil pressure supercharging process; The oil under the action of pressure difference through the damping hole into the process of the accumulator; The process of adding pressure accumulator hydraulic oil volume. So can be structurally pulley as buffer device is composed of four subsystems: the hydraulic cylinder force balance subsystem; The hydraulic cylinder oil chamber flow continuous subsystem; Damping orifice subsystem; Accumulator subsystem.

Four subsystems of the hydraulic system for single output ${ }^{[7]}$, so you can chain using four function neurons respectively simulated four subsystems, the different structure parameters of the system at the beginning of the impact of the same quality and speed of response as a sample set. Of four neurons under different structure parameters of training samples, know that can very good approximation the training sample. According to the function block buffer device's structure and the interaction relationship between subsystems, four subsystems corresponding model of neurons connected, form the corresponding neural network model.

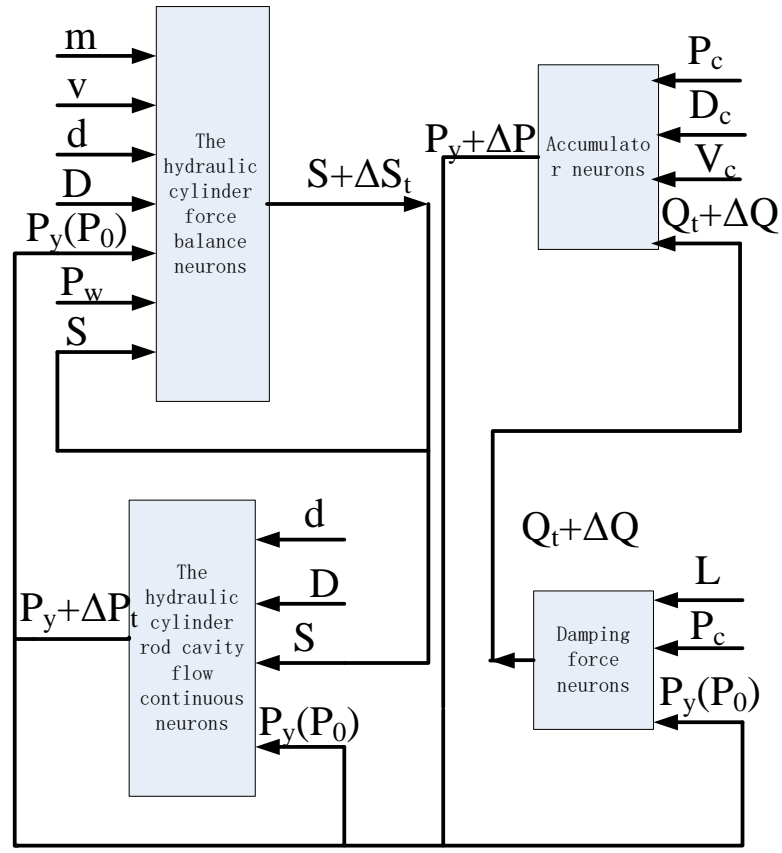

Fig. 2 The model of neural network based on system architecture of sheave buffer Hydraulic buffer is used evaluation method of buffer efficiency: $\eta=\frac{Q}{F_{\max } S}, Q$ is hydraulic buffer to absorb energy, $F_{\text {max }}$ is maximum damping force for hydraulic buffer, $S$ is the hydraulic cylinder piston stroke, hydraulic buffer efficiency as the evaluation index of block buffer. 


\section{The Simulation and Analysis}

Neurons respectively with the improved Sigmoid function as the excitation function, the different structure parameters of the system under the impact of the same quality and speed of response as sample set, sample curve obtained by the AMEsim software simulation, and through standardization, make its output value in the range of neurons, using vector containing dynamic BP algorithm respectively for the above four subsystems function chain neural model and buffers to neurons for training, until each neuron model can well simulate the corresponding subsystem.

BP neural network model code for ${ }^{[8]}$ :

clear all;\% Clear all variables

net =newff(minmax(P),[m,n],\{ 'tansig','purelin','traingdm'\};\% Create network and initialized net.trainparam.show $=100 ; \%$ Interval training status display

net.trainparam.lr=0.01;\% Learning step

net.trainparam.epochs $=500 ; \%$ The simulation number

net.trainparam.goal=1e3;\% The target accuracy

[net,tr]=train(net,P,T);\% Network training

$\mathrm{Y}=\operatorname{sim}($ net, $\mathrm{Q}) \%$ To evaluate the simulation calculation parameter matrix

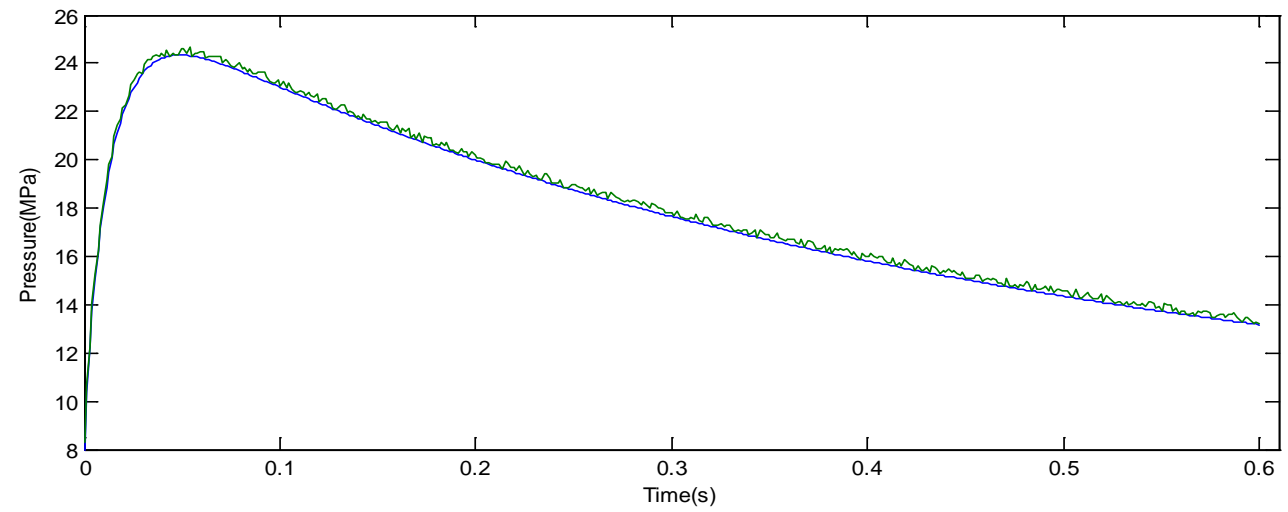

Fig. 3 sheave buffer rod port pressure

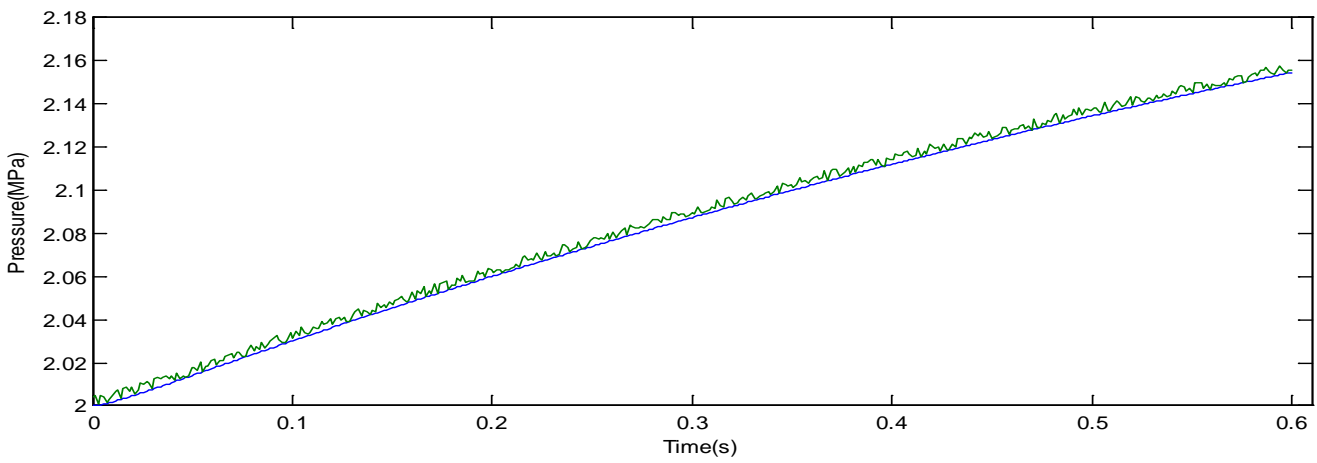

Fig. 4 sheave buffer accumulator pressure

Hydraulic buffer system model as shown in the above test results, it can be seen that the system model of variable output value and output close to expectations. Through corresponding test matrix substitution at the same time, the simulation error is $1.47 \%$, it said in the input parameter is the significance of the combination of the BP neural network model with output close to the results of actual model, based on the structure of the neural network model has better approximation ability.

\section{Summary}

Neural network modeling method based on the structure of the model, the dynamic process of the pulley buffer can get more accurate dynamic model, as well as through the method for structural parameters optimization of the subsequent pulley buffer and pulley buffer system lay a good foundation for the optimization design. 


\section{References}

[1]. Chengbang Zhu. Modern carrier arresting gear. Shipboard Weapons. 2009, (10):75-79.

[2]. Xun.Ou. Carrier arresting gear. The Modern Ship.2005, (9): 45-47.

[3]. Haidong Wang, Bing heng Yang, Yuquan Bi, Kui Huang.Dynamic analysis of MK7-3 type arresting device buffer system. Ship science and technology.2011,33 (3) :141-143.

[4]. Haiwei Yang, Yongqi Zhan, Jun-wei Qiao, Guanglin Shi. Application in Structural Paramater Optimization by using Architecture Based Neural Network[J]. Journal of System Simulation,2003, 15(5):1116-1119.

[5]. Pingfan Yan, Changshui Zhang. Artificial neural network and simulated evolutionary computation.Beijing:Tsinghua University Press,2001

[6]. Jun-wei Qiao, Yongqi Zhan, Guanglin Shi.Research on modeling and optimizing method employing like neural networks based on system architecture[J].Chinese Journal of Mechanical Engineering,2002,38(1):71-74.

[7]. Junwei Qiao, Yong-qi Zhan, Guanglin Shi.Application of neural networks based on system ar-chitecture in nonlinear modeling[J].Journal of System Simulation,2002,14(5):557-561.

[8]. Xiang Li, Yingjie Yang, Qianyu Wang, Xiaopeng Du. Study on Evaluation Model of Maintenance Staff Grouping Based on BP Neural Network in Wartime[J]. Computer \& Digital Engineering.2011, 39(8):25-28,42. 